
METHODS

\title{
On the Inverse Problem of Magnetostatics
}

\author{
M. L. Shur ${ }^{a}$, A. P. Novoslugina ${ }^{b}$, and Ya. G. Smorodinskii ${ }^{b}$ \\ ${ }^{a}$ Ural Federal University, Yekaterinburg, Russia \\ ${ }^{b}$ Institute of Physics of Metals, Ural Branch, Russian Academy of Sciences, Yekaterinburg, Russia \\ e-mails:msh@sky.ru; novoslugina@imp.uran.ru; sm@imp.uran.ru
}

Recieved December 19, 2012

\begin{abstract}
This work is devoted to solving the inverse problem of the magnetic method for nondestructive testing (MMNDT). The purpose of the work, frankly speaking and avoiding complicated concepts and formulas, is to identify research directions in MMNDT that would approach solution of the inverse problem in the field of magnetic defectoscopy to the highest extent.
\end{abstract}

Keywords: magnetostatics, magnetic method, inverse problem, magnetic flux leakage field

DOI: $10.1134 / \mathrm{S} 106183091308007 \mathrm{X}$

\section{INTRODUCTION}

Information technologies and computers with different software packages, by means of which it is possible to solve many problems connected with the calculation of an electromagnetic field for specified initial and boundary conditions, have rapidly penetrated in modern science. Further development of magnetic methods for nondestructive testing (MMNDT) is certainly associated with new computer software packages and contemporary methods for processing measurement results. It is necessary to use the maximum of these possibilities for determining the geometric parameters of a defect via a measured magnetostatic field.

It is an urgent task to solve the inverse problem of magnetostatics. It would be ideal to learn how to determine the parameters of a defect, i.e., the length, depth and width, and depth of defect occurrence, with respect to the surface of a manufactured article, via a field measured at multiple points outside the monitored product.

In general terms, the field of a magnetized ferromagnetic magnet can be represented as

$$
\mathbf{H}(\mathbf{r})=-\operatorname{grad} \varphi(\mathbf{r})=-\frac{1}{4 \pi} \operatorname{grad} \iiint_{V_{0}} \frac{\mathbf{M}\left(\mathbf{r}_{0}\right)\left(\mathbf{r}-\mathbf{r}_{0}\right)}{\left|\mathbf{r}-\mathbf{r}_{0}\right|^{3}} d V_{0} .
$$

When magnetization at the point $r_{0}$ depends unequivocally on a field at the same point, the direct problem is described by Eq. (1) and has one unknown quantity, that is, the field, since the surface that bounds the volume of a ferromagnetic material is known. However, only a field that is measured out of a ferromagnet is known when solving the inverse problem, but the shape of a defect and its geometric parameters are unknown. Hence, Eq. (1) has two unknowns, i.e., the field and defect surface. Therefore, the inverse problem in this formulation cannot be solved. This issue will be addressed in detail in the next section of this paper.

A large number of studies [1-15] have been devoted to solving the inverse problem. Numerical analysis of the magnetic field configurations of the ferromagnetic objects of limited length, which have surface and subsurface defects, was performed in $[12,13]$ by the method of spatial integral equations. As claimed by the authors, that results that are obtained by this method can be used in solving inverse problems of defectoscopy. To determine the parameters of defects, the authors of [14] made use of systems of regression equations and attempted to increase the accuracy in the estimation of defect sizes by dividing the set of defects into subclasses. However, the fundamentally important issue of the formulation of the inverse problem was discussed in none of the above works. For this reason it is expedient to examine the formulation, issues, and solutions of the inverse problem. 


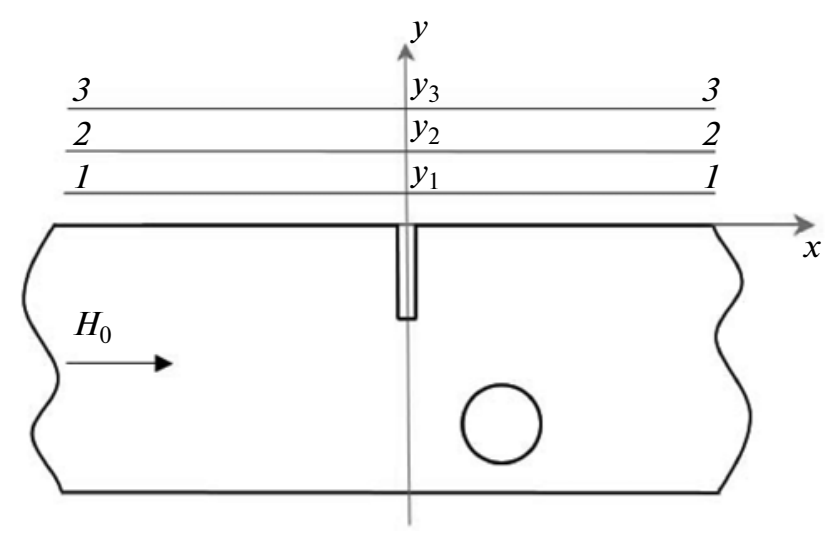

Fig. 1. A plane-parallel plate.

\section{DIRECT AND INVERSE PROBLEMS}

Direct problems always describe cause-and-effect relationships. The accuracy of this description depends on the accuracy and depth of comprehension of the physics of running processes. The direct problem can be written as the following equation:

$$
A z(s, x)=u(x),
$$

where $A$ is some known operator, for example, the integral with respect to the variable $s ; z(s, x)$ is a known function; and $u(x)$ is a function that should be calculated.

The equation of the inverse problem is also of the form (2), but $u(x)$ is a known function in it and $z(s$, $x$ ) is an unknown function that should be calculated.

Nominally, an equation of the inverse problem can be written in the form

$$
z(x)=A^{-1} u(x)
$$

where $A^{-1}$ is the inverse operator of $A$.

It may seem that these two formulations are completely equivalent, but they are not. Let $A$ be the differential operator $d / d x$. No matter how complicated the function $f(x)$ is, we always can find its derivative. The operator inverse to the operator of differentiation is an integral, which is not always possible to calculate by any means. This paradigm demonstrates the pure computational difference between the direct and inverse problems, but they are both correct in the given example.

The incorrectness of inverse problems that are considered in geophysics and other areas of physics is connected, first of all, with the inaccuracy of the measurements and unsteadiness of the solution. Even small inaccuracies in measuring the values that are associated with the presence of noise, can give rise, for example, to very large differences in a solution. This may lead to the fact that the result will not have anything to do with reality.

The inverse problem of MMNDT is more complicated. It is described by Eq. (1), in which the magnetization is unknown not only inside the product but also in its surface, since it is unknown, which type of defect, viz., surface or internal defects, create the measured field. Moreover, precisely the shape of the product and, namely, the size of the defect are of interest. Therefore the inverse problem of MMNDT is described by the following equation:

$$
A z(s, x)=u(x),
$$

where $A$ is some unknown operator, whose form depends on the form of a ferromagnetic surface; $u(x)$ is a known function measured in a finite, albeit in a very large number of points; and $z(s, x)$ is an unknown function that must be calculated.

As was already indicated above, this kind of a problem, as well as an equation with two unknowns, generally has an infinite set of solutions. One can tell from the viewpoint of MMNDT that this problem does not have a solution at all.

To understand the consequences of this difficulty, which is fundamental in terms of mathematics, let us examine Fig. 1, which illustrates the monitored product in the form of a plane-parallel plate with defects. Magnetic-field measurements are carried out along the line 1-1. Suppose that measurements of 


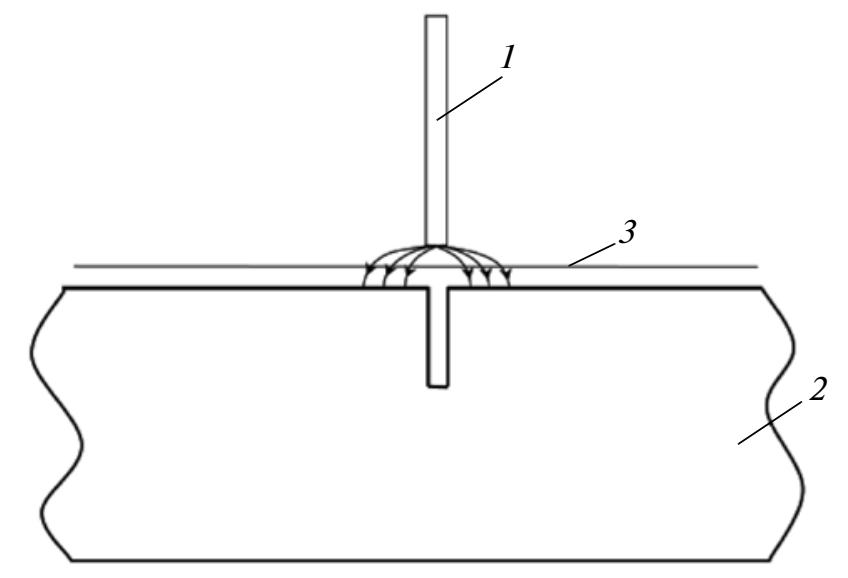

Fig. 2. The scheme of the monitoring: 1 is a magnet, 2 is a plate with a surface defect, and 3 is the line of monitoring.

the normal component of the field are carried out in an arbitrarily large number of points with unrestrictedly high accuracy. It is known from mathematical physics that knowing the field at infinity, which is equal to the magnetization field $H_{0}$, one can calculate the field, i.e., any of its components, in the entire space above the line $1-1$. This field will be the same if dipoles with a dipole moment of $d_{1}=H_{y}\left(x, y_{1}\right)$ are arranged on the line $1-1$, but it is impossible to calculate the field below the line $1-1$, since the field at the boundary of the monitored product is unknown.

If the $H_{y}$-component of the field is measured along the line 2-2, then the field above this line can be calculated. It will be the same as if dipoles with a dipole moment of $d_{2}=H_{y}\left(x, y_{2}\right)$ are arranged on the line $2-2$. The fields of the dipole $d_{1}$ and $d_{2}$ will be absolutely equal in all the points above the line $2-2$. We are faced, apparently, with a very simple problem. We know that the field is created either by the dipoles $d_{1}$ or dipoles $d_{2}$. The dipole moment value of these dipoles is known. The problem is to determine by which dipole, $d_{1}$ or $d_{2}$, it is created by measuring the field along the line $3-3$. As follows from the above, it is impossible to determine. This kind of an inverse problem has no solution and is totally incorrect. In order to formulate the problem of MMNDT correctly, it is necessary to make some additional assumptions.

\section{SURFACE MONITORING}

The inverse problem for monitoring the surface of a ferromagnetic item was formulated in [7-9]. To the best of our knowledge, these are the only works in which the inverse problem of MMNDT is really formulated.

We offer another formulation of the inverse problem for monitoring the surface of a ferromagnetic item, which allows one to avoid the problems that were described in Section 1. This formulation will be written for a two-dimensional case. To generalize it to three dimensions is not difficult.

Technically, surface monitoring is much simpler than monitoring an entire manufactured article. This is due to the fact that the need for magnetization of the entire article is avoided; the use of attached magnets that always weigh hundreds of pounds, consume large currents, and significantly increase the cost of a defectoscope system complicates the monitoring procedure. It is much easier to monitor the surface by the scheme shown in Fig. 2.

Due to the fact that the field inside an unsaturated ferromagnet is proportional to $1 / \mu$, and at the same time the absolute magnetic permeability is high $(\mu \approx 1000)$, it can be argued that the magnetic field inside the ferromagnet is very low. On account of the continuity of the tangential component of the magnetic field at the boundary, it will be close to zero on the entire ferromagnetic surface. The continuous tangential component of the magnetic field means that the surface of an item is equipotential, i.e., it has a constant magnetic potential. Since the potential is determined with an accuracy that lies within a constant value, then

$$
\varphi=\varphi_{0}+\varphi_{L}=0, \text { or } \varphi_{L}=-\varphi_{0}
$$

where $\varphi$ is the full potential of the magnetic field produced by the magnetizing device and magnetization of the ferromagnetic material; $\varphi_{0}$ is the potential of the magnetizing device, which is known; and $\varphi_{L}$ is the potential created by magnetization of the ferromagnet. 
Let us assume that the equation of the ferromagnet boundary, $y_{1}=y_{1}\left(x_{1}\right)$, is identified. In this case, it is possible to reproduce the potential $\varphi_{L}(x, y)$ in the entire space outside the ferromagnet via the known potential $\varphi_{L}$ on the boundary. This problem is called the Dirichlet problem; it is expressed by the following formula:

$$
\varphi_{L}(x, y)=-\frac{1}{2 \pi} \int_{L} \varphi_{0}\left(x_{1}, y_{1}\left(x_{1}\right)\right) \frac{\partial}{\partial n_{1}}\left[\ln \left(x-x_{1}\right)^{2}+\left(y-y_{1}(x)\right)^{2}\right] d l
$$

The derivative with respect to the surface normal is expressed as follows: $\frac{\partial}{\partial n_{1}}=\cos \alpha \frac{\partial}{\partial x_{1}}+\sin \alpha \frac{\partial}{\partial y_{1}}$; $\cos \alpha=\frac{1}{\left(1+y^{\prime 2}\right)^{1 / 2}}, \sin \alpha=\frac{y^{\prime}}{\left(1+y^{\prime 2}\right)^{1 / 2}} ;$ and $d l=\left(1+y^{\prime 2}\right)^{1 / 2} d x_{1}$. After applying these relationships, Eq. (6) for values of the potential $\varphi_{L}\left(x, y_{0}\right)$ on the line of measurement, $y=y_{0}$, takes the following form:

$$
\begin{aligned}
\varphi_{L}\left(x, y_{0}\right)= & -\frac{1}{2 \pi} \int_{-\infty}^{\infty}\left\{\varphi _ { 0 } ( x _ { 1 } , y _ { 1 } ( x ) ) \left[\frac{x-x_{1}}{\left(x-x_{1}\right)^{2}+\left(\left(y_{0}-y_{1}\right)(x)\right)^{2}}\right.\right. \\
& \left.\left.-y^{\prime}\left(x_{1}\right) \frac{y_{0}-y_{1}(x)}{\left(x-x_{1}\right)^{2}+\left(y_{0}-y_{1}(x)\right)^{2}}\right] d x_{1}\right\} .
\end{aligned}
$$

In practice, it is not the potential that is measured but rather one of the components of a magnetic field, whose value can be obtained by differentiating Eq. (7) with respect to the $x$ or $y$ coordinate. The formulas that are derived from differentiation are not given in this paper, since our goal was to show how the inverse problem of establishing the presence of a defect and determining its dimensions for a particular case of the item surface monitoring can be reduced to well-studied problems in geophysics that are solved using nonlinear integral equations.

As a result we obtain a nonlinear integral equation in the form

$$
u\left(x, y_{0}\right)=u_{0}\left(x, y_{0}\right)+\int_{a}^{b} \varphi_{0}\left(x_{1}, y_{1}\left(x_{1}\right), y_{0}\right) K\left(x_{1}, y_{1}\left(x_{1}\right), x, y_{0}\right) d x_{1},
$$

where $u\left(x, y_{0}\right)$ is the $x$ - or $y$-component of the magnetic field measured on the line $y=y_{0} ; u_{0}\left(x, y_{0}\right)$ is the $x$-or $y$-component of the magnetic field created by the magnetizing device; and $K(\ldots)$ is a known function.

This integral equation is more complex than those that arise in geophysics, since there is a rather complicated function, $\varphi_{0}$, under the integral, which equals a constant in problems of geophysics. However, the general methods for the solution of incorrect inverse problems, which are described by nonlinear integral equations, are also applicable to Eq. (8) as well.

Thus, we have redefined the problem of determining the geometric parameters of surface defects by using the additional statement that the magnetic potential on the surface of a ferromagnetic item or the tangential component of a magnetic field equals zero. This allowed us to obtain Eq. (8).

\section{ON THE REDEFINITION OF THE INVERSE PROBLEM OF MAGNETOSTATICS FOR SOME DEFECT CLASSES}

The incorrect inverse problem of magnetostatics can be redefined only on the ground of physical considerations derived from a deep understanding of physical principles of the formation of defect fields in ferromagnetic materials, whose magnetic permeability (magnetic induction and magnetization) nonlinearly depends on the magnetic field.

Taking the nonlinear properties of ferromagnetic materials into account is fundamentally important. For example, the Zatsepin-Shcherbinin formula, which describes the fields of defects that appear in the form of a rectangular groove with a large degree of accuracy, is well known in the theory of magnetic fields. This formula is derived on the basis of the assumption that the magnetization is constant along the lateral faces of a defect. It was shown in [16-17] that this is true, but primarily due to the nonlinear dependence of the magnetic permeability on the magnetic field. 
Let us consider the problem of determining the radius and location of a defect in the form of an infinitely long cylinder with a circular cross section. This is the simplest problem from the standpoint of the MMNDT requirements. The inverse problem allows one to understand issues that arise when solving this kind of problem.

The magnetic field, $\mathbf{H}$, in the linear ferromagnetic medium is described by the equation

$$
\operatorname{div} \mathbf{H}=0 .
$$

The solution of this equation for an infinitely long cylinder with the circular section appears in the form

$$
\mathbf{H}=\mathbf{H}_{0}+\mathbf{H}_{0} a^{2} f(x, y),
$$

where $a$ is the radius of a defect and $f(x, y)$ is a function that describes the dependence of a field on the coordinates for a dipole that is located at the origin of coordinates.

By measuring the magnetic field at three points, we obtain a system of three nonlinear algebraic equations for the three unknowns, $a, x$, and $y$, from which the radius and coordinates of a defect can be determined unambiguously. The latter statement about unambiguous determination follows from the physical meaning of these equations. In fact, a quadratic equation with one unknown, for example, has two solutions.

The magnetic field, $\mathbf{H}$, in a nonlinear ferromagnetic medium is described by the equation with a nonzero right side,

$$
\operatorname{div} \mathbf{H}=-\operatorname{div} \mathbf{M} .
$$

By analogy with electrostatics, the quantity-divM is called the volume magnetic charge.

Our theoretical studies, which are not presented here, allow one to conclude that the nonlinearity of a real ferromagnetic medium does not change the dipole nature of the field of a cylindrical defect with a circular cross section, which can be described by the following function:

$$
\mathbf{H}_{d} \sim \mathbf{H}_{0}^{\prime} a^{\prime 2} f(x, y),
$$

where $\mathbf{H}_{0}^{\prime}$ is some effective field and $a^{\prime}$ is the radius of a defect, whose value depends on the magnetization curve of a particular ferromagnet.

Therefore, by measuring the field in three points and solving the system of three nonlinear algebraic equations, we can determine the effective radius of a defect. To determine its real radius, one needs to know the functional dependence of the effective radius on the external field for this particular ferromagnet. To determine this relationship, it is necessary to solve Eq. (11), i.e., to solve the direct problem.

Let us assume that this was successfully done. Then, the problem of determining the radius of a defect and its location in terms of mathematics is reduced to a system of three nonlinear algebraic equations with three unknowns. Thus, by reducing the inverse problem of determining the geometric parameters of a defect in the form of an infinitely long cylinder with a circular cross section to Eq. (12), we have accomplished the redefinition.

However, this task is still incorrect for all ordinary purposes, since measurement errors can significantly affect the accuracy of the values that are obtained as a result of calculations. Let us explain this issue by giving the example of solving the following quadratic equation with one unknown:

$$
a x^{2}+b x+c=0 .
$$

The coefficients $a, b$, and $c$ in this equation are determined experimentally with some error. Let the true values of these coefficients be such that the discriminant $d=b^{2}-4 a c=0$. Obviously, even a small error of measurement may lead to the case where the discriminant value $d<0$, i.e., to the conclusion that there is no solution at all.

Thus, even the simplest MMNDT inverse problem of determining the geometric parameters of an infinitely long cylinder with a circular cross section in a linear ferromagnetic medium, which is described by Eq. (10), is incorrect and requires the use of a complex mathematical apparatus for its solutions. In reality, the problem of determining the location and magnitude of a dipole moment is related to the magnetic location of submarines or metal objects in a human body. An extensive literature is devoted to this problem, but the ultimate solution has still not been found. But is there any need to follow this path? Is there any demand for solving the inverse problem? 


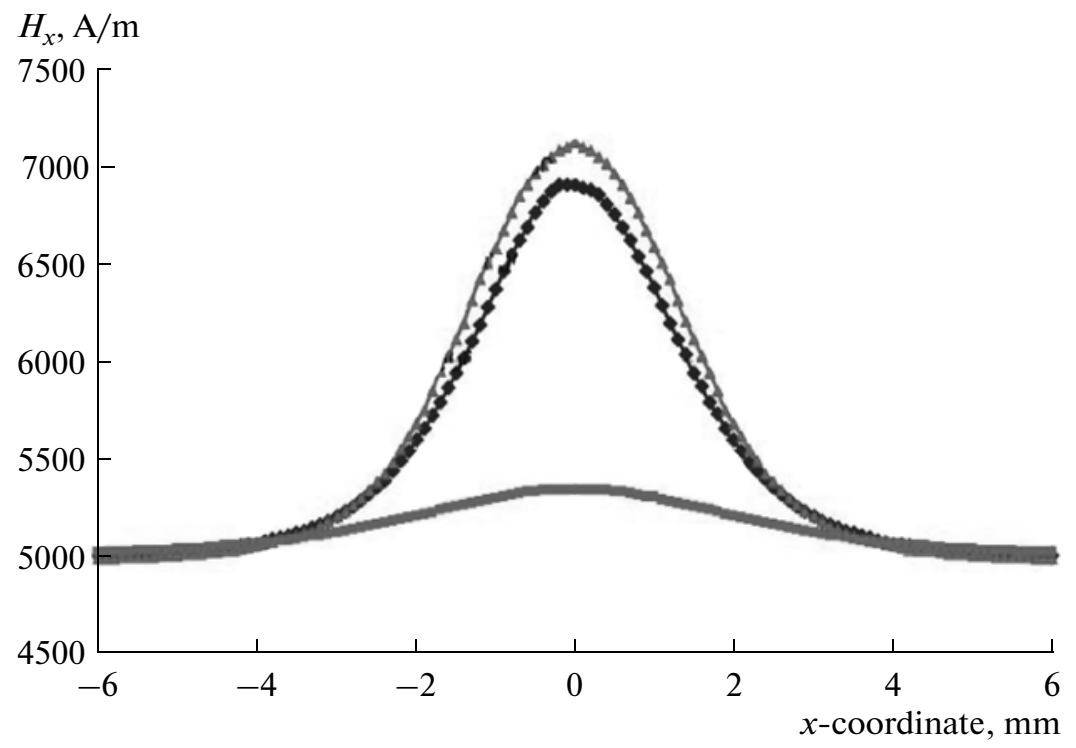

Fig. 3. The $H_{x}$-component of the magnetic field of a defect in a manufactured article of different steel grades, as follows: $\longrightarrow$ is for 20 ;

$\longrightarrow$ is for 30ХГСА;

$\_$is for 10 .

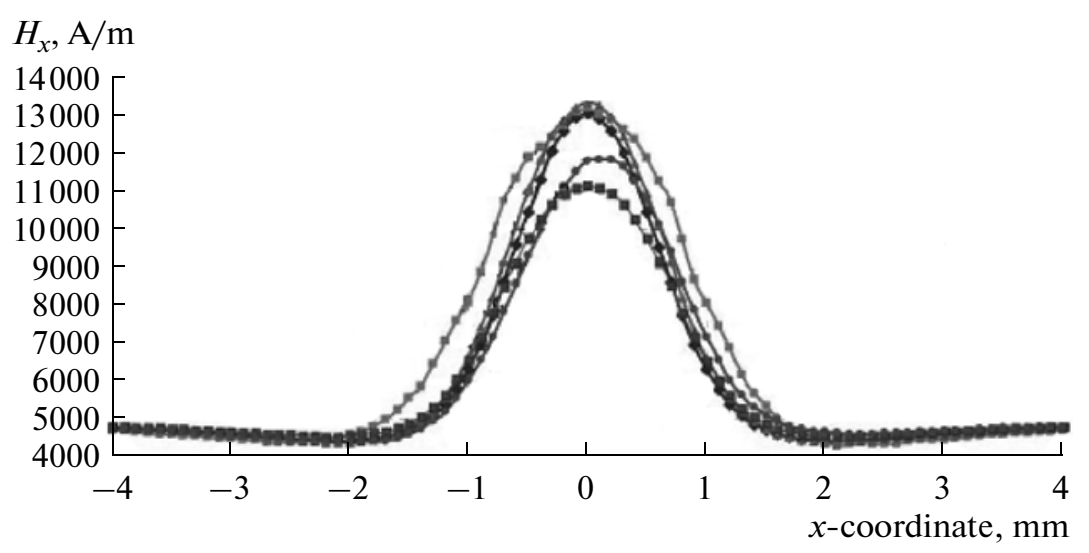

Fig. 4. The $H_{x}$-component of the magnetic field for different types of a defect cross section, as follows:

$\longrightarrow$ is for a circle;

$\longrightarrow$ is for a square;

$\longrightarrow$ is for a hexagon;

$x-$ is for a triangle;

$\longrightarrow$ is for a polygon.

\section{A PHENOMENOLOGICAL APPROACH TO SOLVING THE INVERSE PROBLEM}

As a consequence of the above discussion, an important conclusion can be made that the inverse problem is possible to solve only on the basis of careful study of the direct problem and, most importantly, on the basis of a deep understanding of the physics of the process under investigation. This statement is not new; it is well-known to geoscientists and is apparent to experts in the field of MMNDT.

Experimental and theoretical studies on field defects were reported in [16-21]. Unfortunately, all the significant results in this area date back to the last century. It is difficult to pick out any noteworthy results that were obtained in the last 20 years. 


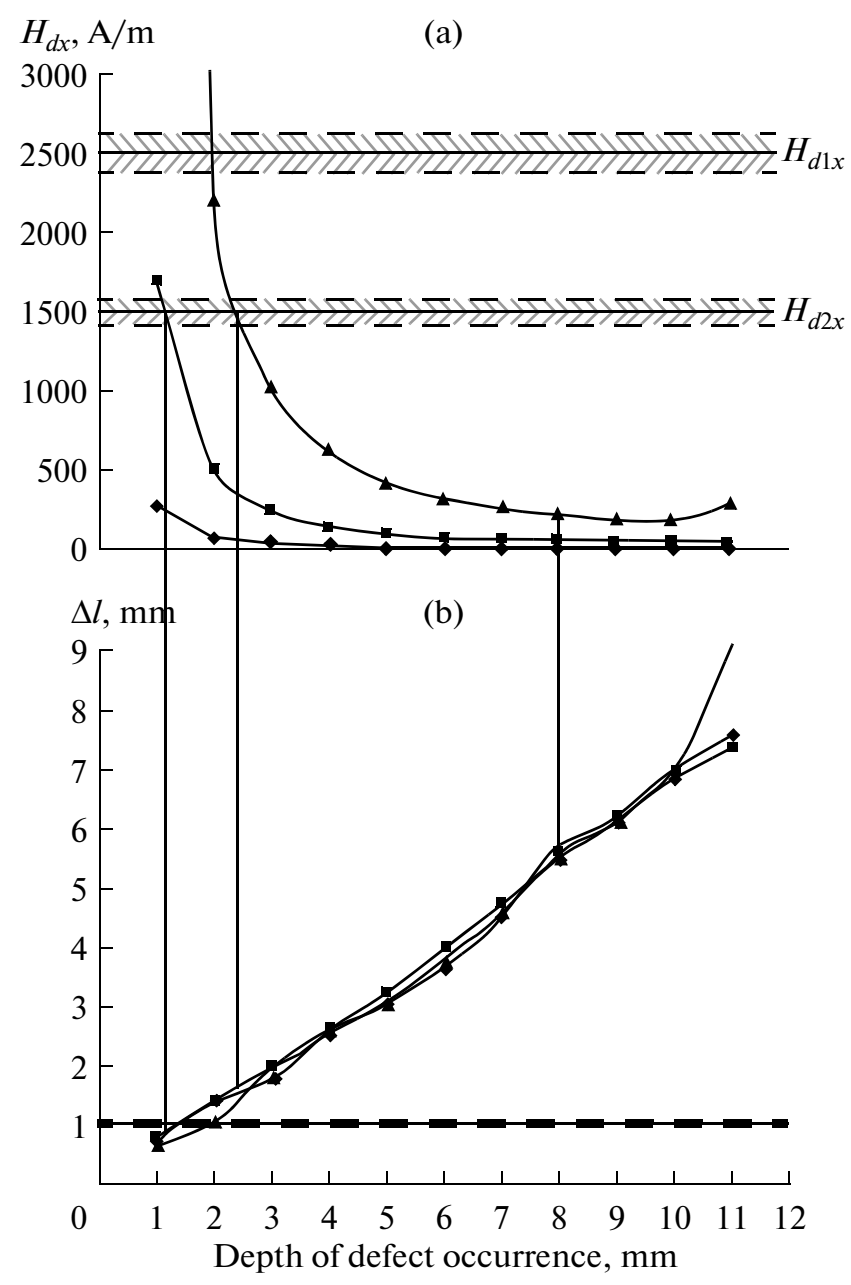

Fig. 5. The determination of defect parameters, that is: the dependence of the maximal value of the field $x$-component on the of depth of defect occurrence for defects with various radii (a); the dependence of the distance between extremes of the magnetic field $y$-component on the defect depth level (b); designation of lines by radii:

$\longrightarrow$ is for 0.2 ;

- is for 0.5 ;

$\longrightarrow$ is for 1.0 .

Modern computers and existing software packages allow one to calculate the fields of real defects in real ferromagnets with a high level of accuracy. Based on the results we have obtained, some important conclusions can be made regarding a solution of the inverse problem of MMNDT.

The dependence graphs of the $x$-component of the field of a cylindrical defect with a circular cross section on the coordinate $x$ for a plane-parallel plate are shown in Fig. 3; evidently, the magnetic field depends on the properties of the ferromagnetic material. The dependence curves of the $H_{x}$-component of the magnetic field of a defect with a cross section in the form of a circle, square, hexagon, triangle, and polygon on the $x$ coordinate are given in Fig. 4. The defect fields are very close to each other, so it is unlikely that the types of defects can be distinguished in the process of monitoring the object.

The three dependence curves of the maximal value of the field $x$-component on the depth of defect occurrence for defects with various radii are given in Fig. 5a. Since vibrations between an item and magnetizing device, between an item and sensor, as well as other noises of different physical natures, always occur in the monitoring process, then the measured field of a defect differs from the true field and lies within some interval. For the sake of certainty, let us assume that this random deviation does not exceed $\pm 5 \%$. Let the average measured value be equal to $H_{d 1 x}$ (Fig. 5a). It is safe to say in this case that the radius of a defect equals $1 \mathrm{~mm}$ and the defect depth level can be determined rather accurately. 
If the mean value of the measured field equals $H_{d 2 x}$, then the problem becomes more complicated. The radius of a defect in this case cannot be determined via the measured field. It can be either 1 or $0.5 \mathrm{~mm}$. In order to determine the radius for this case, let us introduce an additional parameter, that is, the distance between the extremes of the $y$-component of a magnetic field. The dependence of the distance between the extremes of the magnetic field of the $y$-component, $\Delta l$, on the depth of defect occurrence is given in Fig. 5b. The fact that $\Delta l$ almost does not depend on the defect radius but is sensitive to the depth of defect occurrence seems to be important. The depth of defect occurrence is determined from Fig. 5b as follows: if it equals $1.2 \mathrm{~mm}$, then the defect radius is $0.5 \mathrm{~mm}$; if it equals $2.4 \mathrm{~mm}$, then the defect radius is $1 \mathrm{~mm}$.

At greater depths of defect occurrence, the problem is not in discriminating a defect with a radius of $0.5 \mathrm{~mm}$ from a defect with a radius of $0.2 \mathrm{~mm}$, the question is whether it is possible to reveal these defects. However, a defect with a diameter of $1 \mathrm{~mm}$, is definitely unsafe and the item should be rejected as defective, while the defect with a diameter of $0.4 \mathrm{~mm}$ may be acceptable.

Analyzing Figs. 5a and 5b, the following conclusions can be drawn:

1. Using the parameter $\Delta l$, the depth of defect occurrence can be determined quite accurately, even at a measurement accuracy of $\pm 20 \%$, but it is impossible to determine the radius of a defect in this case.

2. The use of the two parameters, $H_{d x}$ and $\Delta l$, allows one to determine the defect radius more precisely, which is one of the main rejection parameters in a manufacturing site.

3. There are difficulties in determining the radii of defects at greater depths of defect occurrence.

\section{CONCLUSIONS}

The possibility of solving the inverse problem with the aid of mathematical tools, such as those that have been developed for the purpose of geophysics, has been considered. Based on the results that were reported in the present work, it can be argued that the inverse problem does not have a solution in such a generalized statement as in Eq. (1), since, in order to determine the defect shape, it is necessary to know the magnetization distribution inside a manufactured article, which in turn depends on the defect shape.

If the defect in an item is superficial, then a mathematical solution of the inverse problem is possible, since in this case the surface of the item with a defect is known.

For internal defects, in our opinion, the only possible way to solve the inverse problem is the following:

To partition all of the defects into classes;

To find the characteristic attributes for each class, which would define belonging to a certain class; and

To determine the geometric parameters of typical defects from the selected classes by comparing the measured field with the calculated field.

This approach has been demonstrated in this work based on the example of an elongated defect with a cross section that is close to a circle. Indeed, for the rejection of a manufactured article as defective, often it does not matter whether the defect cross section is a circle or square. It is important to know which part of the working body of an item has failed. It is easy to perform similar analysis of the magnetic field for defects with the spherical and close-to-spherical shapes. By partitioning the defects into classes, a database with values of the defect field parameters can be formed, which would allow one to determine the approximate shape of a defect and its dimensions with an accuracy that does not exceed $10 \%$ of the item thickness.

\section{ACKNOWLEDGMENTS}

This work was supported by the program of the Presidium of the Russian Academy of Sciences (grant no. 12-P-1031-2)

\section{REFERENCES}

1. Yan, M., Udpa, S., Mandayam, S., Sun, Y., Sacks, P., and Lord, W., Solution of inverse problems in electromagnetic NDE using finite element methods, IEEE Trans. Magn., 1998, vol. 34, no. 5, pp. 2924-2927.

2. Chadebec, O., Coulomb, J.-L., Bongiraud, J.L., Cauffet, G., and Thiec, P., Recent improvements for solving inverse magnetostatic problems applied to thin shells, IEEE Trans. Magn., 2002, vol. 38, no. 2, pp. 1005-1008.

3. Vuillermet, Y., Chadebec, O., Coulomb, J.-L., Rouve, L.-L., Cauffet, G., Bongiraud, J.L., and Demilier, L., Scalar potential formulation and inverse problem applied to thin magnetic sheets, IEEE Trans. Magn., 2008, vol. 44, no. 6, pp. 1054-1057. 
4. Chadebec, O., Coulomb, J.-L., and Janet, F., A review of magnetostatic moment method, IEEE Trans. Magn., 2006, vol. 42, no. 4, pp. 515-520.

5. Guerin, S., Cauffet, G., Coulomb, J.-L., and Bongiraud, J.L., Robustness of a Magnetostatic Inverse Problem Resolution. Marelec, 2006.

6. Zhirkov, V.F., Novikov, K.V., and Sushkova, L.T., Solution of the inverse problem of magnetostatics by Tikhonov's regularization method, Doklady 7-i mezhdunarodnoi konferentsii DSPA (Proc. 7th Int. Conference DSPA), 2005, vol. 1, p. 2005.

7. Cherepanov, A.A., Krotova, E.L., and Krotov, L.N., Statement and solution of the inverse geometric problem of magnetostatics for magnetic defectoscopy, Tezisy X Vserossiiskogo simpoziuma po prikladnoi i promyshlennoi matematike (osennyaya otkrytaya sessiya) (Proc. 10th All-Russian Workshop on Applied and Industrial Mathematics, Open Session), Sochi-Dagomys, 2009.

8. Krotov, L.N., Reconstruction of a media boundary based on the spatial distribution of stray magnetic fields. II. Definition and method of solving the inverse geometric problem of magnetostatics, Russ. J. Nondestr. Test., 2004, vol. 40, no. 6, pp. 385-390.

9. Krotov, L.N., Mel'nik, R.S., and Yakovlev, M.V., Approximate recovery of the surface defect form of welded joint by the magnetic method, Doklady Vserossiiskoi s mezhdunarodnym uchastiem nauchno-tekhnicheskoi konferentsii "Svarka i kontrol'-2004" (Proc. All-Russian Science and Technical Conference with Intern. Attendance 'Welding and Monitoring-2004'), Perm, 2004, vol. 2, pp. 318-325.

10. Dyakin, V.V., Direct and inverse problem of magnetostatics, Defektoskopiya, 1996, no. 3, pp. 3-6.

11. Karpov, R.G., Diagnostic system of the magnetic field sources, Desyataya Vserossiiskaya mezhvuzovskaya nauchno-tekhnicheskaya konferentsiya studentov $i$ aspirantov (Proc. 10th Al-Russian Interuniversity Science and Technical Conference for Undergraduate and Graduate Students), Moscow: MIET, 2003, p. 198.

12. Gal'chenko, V.Ya., Ostapushchenko, D.L., and Vorob'ev, M.A., Computer analysis of the configuration of the magnetic fields of surface discontinuity flaws of finite dimensions in a ferromagnetic plate of finite dimensions by the spatial integral equation method, Russ. J. Nondestr. Test., 2009, vol. 45, no. 3, pp. 191-198.

13. Gal'chenko, V.Ya., Ostapushchenko, D.L., and Vorob'ev, M.A., Computer Analysis of the Configurations of magnetic fields of subsurface discontinuities of finite dimensions and arbitrary shapes in tested objects of finite length by the method of spatial integral equations, Russ. J. Nondestr. Test., 2009, vol. 45, no. 5, pp. 337-346.

14. Slesarev, D.A., Barat, V.A., and Chobanu, P.M., A decrease in the error of the statistical method for estimating the parameters of flaws in magnetic flaw detection, Russ. J. Nondestr. Test., 2012, vol. 48, no. 1, pp. 55-48.

15. Muzhitskii, V.F. and Efimov, A.G., A model of a surface flaw of finite length under normal magnetization and calculation of the topography of its magnetostatic field, Russ. J. Nondestr. Test., 2008, vol. 44, no. 3, pp. 167-177.

16. Zatsepin, N.N. and Shcherbinin, V.E., Towards calculation of a magnetostatic field of the surface defects. I. Field topography of defect models, Defektoskopiya, 1966, no. 5, pp. 50-58.

17. Shcherbinin, V.E. and Zatsepin, N.N., Towards calculation of a magnetostatic field of the surface defects. II. Experimental verification of main calculated relationships, Defektoskopiya, 1966, no. 5, pp. 59-65.

18. Shcherbinin, V.E., Shur, M.L., and Zagidulin, R.V., Magnetic field topography of a narrow surface defect, Defektoskopiya, 1986, no. 7, pp. 86-88.

19. Shur, M.L., Shcherbinin, V.E., and Zagidulin, R.V., Calculation of a surface defect field in non-linear ferromagnetic medium, Defektoskopiya, 1987, no. 2, pp. 3-9.

20. Shur, M.L., Shcherbinin, V.E., and Zagidulin, R.V., Theoretical issues of the surface defect field generation, Defektoskopiya, 1988, no. 3, pp. 14-25.

21. Ferster, F., Nondestructive examination by the method of stray magnetic fields. Theoretical and experimental grounds of identification of finite- and infinite-depth surface defects, Defektoskopiya, 1982, no. 11, pp. 3-25.

Translated by $O$. Kadkin 